\title{
Hybrid photonic system integration using thin glass platform technology
}

\author{
Henning Schröder $\odot$, a,b,* Julian Schwietering ${ }^{\bullet},{ }^{\text {b }}$ Gunnar Böttger, ${ }^{\text {a }}$ \\ and Vanessa Zamora ${ }^{a, b}$ \\ ${ }^{a}$ Fraunhofer Institute for Reliability and Microintegration (IZM), Berlin, Germany \\ ${ }^{\mathrm{b}}$ Technical University of Berlin, Berlin, Germany
}

\begin{abstract}
Demand for high integration of optoelectronic and micro-optical components into micro-electronic systems for communication, computing, medical, and sensing applications is increasing. Advanced hybrid packaging technologies are used to enhance glass-based substrates featuring electrical, thermal, and optical functionalities with laser diodes, modulators, isolators, photonic integrated circuits (PIC), beam-splitting components, and micro-lenses. Such glassbased substrates can be thin glass layers on large panels or more mini-bench-like boards that can be embedded into organic printed circuit boards (PCBs). Optical fiber interconnects, connectors, and electrical-optical integration platforms are used for higher level system integration and need to be miniaturized on module and board level to fulfill decreasing channel pitch requirements. We provide background on and discuss thin glass as a suitable base material for ion exchanged waveguide panels and interposers, precise glass structuring for posts and holders, the related high precision assembly techniques, and advanced fiber interconnects. Some examples of PCB photonic integration, micro-bench optical sub-assemblies, including PIC, and 3D optical resonator packages that combine most of these approaches will be shown. (C) The Authors. Published by SPIE under a Creative Commons Attribution 4.0 Unported License. Distribution or reproduction of this work in whole or in part requires full attribution of the original publication, including its DOI. [DOI: 10.1117/1.JOM.1.3.033501]
\end{abstract}

Keywords: micro-optics assembly; photonic packaging; fiber alignment; electrical-optical circuit board; system integration; thin glass; optical waveguide; ion exchange; micro-optical bench; whispering gallery bottle resonator.

Paper 21003 received Jan. 18, 2021; accepted for publication Apr. 19, 2021; published online May 14, 2021; corrected Dec. 28, 2021.

\section{Introduction}

An unmet need for photonic packaging services in the worldwide photonics supply chain has just recently begun to be redressed. This gap arose from a lack of interface standardization with the development of new platform technologies and applications, as well as demand for high assembly precision to reach the desired optical coupling efficiency while advancing further miniaturization. For these reasons, the development of photonic system integration now lags some 20 to 30 years behind that of electronic IC packaging. ${ }^{1}$ As shown in Fig. 1, the main technology domains for photonic system integration are fiber optics, planar integrated optics, and photonic assembly technologies. New materials, components, and low loss platform technologies were developed to gain high optical coupling efficiencies, which allow increased miniaturization and complexity for better performance. This is reflected in Fig. 1, showing that progress in terms of tackling the challenges and advancing the technology was driven by the development of specific key applications.

Overall, photonic system integration is primarily driven by an increasing demand for bandwidth in data communication and telecommunication. Higher channel density, fast modulation, and wavelength-division multiplexing are major required functionalities and advancement of these is hard-pressed to keep up with the demand. ${ }^{2}$ In fact, the electrical interconnect bottleneck is expected to be much exacerbated in the near future for chip-to-chip applications in intracomputer communication. ${ }^{3}$ Embedded thin glass layers (sub-mm range) with integrated optical

\footnotetext{
*Address all correspondence to Henning Schröder, henning.schroeder@izm.fraunhofer.de
} 


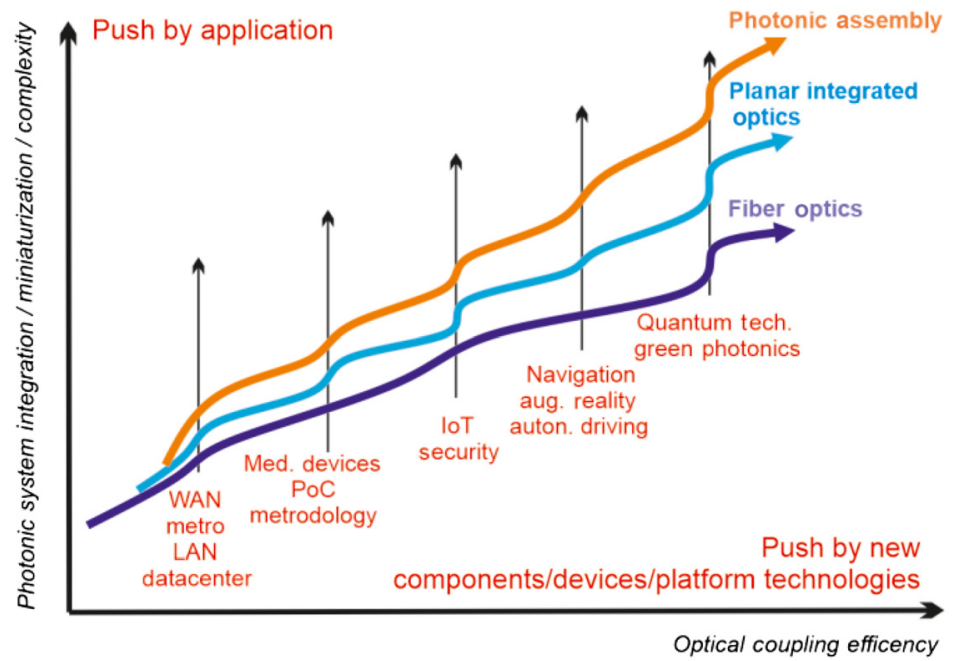

Fig. 1 The main technology domains for photonic system integration (automated photonic assembly, planar integrated optics, and fiber optics) are driven by new applications, components, and platform technologies. Optical coupling efficiency is the most crucial issue in improving miniaturization and complexity.

waveguides are key building blocks to overcome that bandwidth bottleneck. Strong physical arguments exist for prioritizing such optical substrate integration. First, miniaturization requires the transition from extensive, fiber-based optical connectors to substrate integration, a transition comparable to the on-going replacement of discrete electronics, conventional bonding, and sequential wiring schemes with highly integrated and parallelized mounting. An increasing number of sought-after inter- and intra-system optical interconnects cannot be realized by fibers due to packaging cost, weight, density, and handling issues. If connectors remain necessary for plugging purposes, they will need to shrink even in light of higher canal density. Second, photonic packaging has to follow chip and component roadmaps. Apart from vertical-cavity surfaceemitting laser, edge-emitting laser diode (LD), and photodiode (PD) components, photonic integrated circuit (PIC) platforms with integrated active and passive optical functionality also require photonic system integration platforms on higher packaging level. ${ }^{4} \mathrm{PICs}$ are mostly based on silicon-on-isolator (silicon photonics) and silicon nitride material platforms. Typical PIC structures comprise micro- and nano-sized waveguides with strong light confinement, leading to low propagation and bend losses. However, highly efficient and stable optical coupling is required to connect PICs to laser sources and the outside world. Coupling strategies for PICs take two main forms: edge butt coupling and surface coupling (grating, evanescent). Here, edge coupling is the most promising approach, e.g., for the development of compact laser sources, but issues stemming from low tolerance to optical misalignments and thermal effects of glued interfaces have not yet been fully resolved.

PICs typically include internal mode field expansion sections [spot size converter (SSC)] for increasing internal integrated waveguide mode field diameters to a more accessible 2 to $5 \mu \mathrm{m}$ on chip edges. For a desired direct coupling to standard optical fibers without additional focusing elements, this is too small for an acceptable coupling efficiency. For this conversion, custommade additional optical SSC chips with integrated waveguides for increasing mode field diameters to around $10 \mu \mathrm{m}$ are available, fabricated using glass by ion exchange ${ }^{5-7}$ or fs-laser writing. ${ }^{8}$ Tapered structures are employed here, including silicon photonics. ${ }^{9}$ These additional chips typically have more than one waveguide, enabling not only single, but also multiple coupling with mostly 250 - or $125-\mu \mathrm{m}$ pitches. Additionally, internal branching or pitch conversion structures can be included. Integrated conversion structure sizes and minimal waveguide bending radius depend on waveguide technology. As such, external SSC can have very different sizes that can be easily adapted using finely lasered thin glass elements. In particular, U-holders allow gapless gluing onto the substrate by means of the reservoirs and z-shrinkage-free fixing of the SSC chip at the right height. 
Furthermore, glass-based system integration, using thicker glass substrates (mm range) which are highly scalable in size and complexity, is also a promising tool in the miniaturization of more complex and advanced optical systems such as LiDAR, gyroscopes, analytical systems, and a variety of optical sensors. Such optical systems are often well established in optical methodology, as well as emerging application fields (point-of-care, Internet of Things, autonomous driving, and quantum devices) that feature demand for smaller systems and ambitious targets in terms of reliability, volume manufacturing, and cost reduction. Therefore, the thin glass layer integration concept for optical interconnects as proposed below in more detail (Fig. 2) can also be extended to micro- and macro-optical bench-like glass board concepts that include specific assembly components such as mechanical posts, adapters, and holders which are laser-structured from glass, enabling hybrid assemblies with very diverse active components of optical and/or electrical functionality (see Fig. 3).

This paper introduces thin glass as integration and packaging platform for optoelectronic, electronic, and micro-optic components on wafer and panel level. According to the thickness

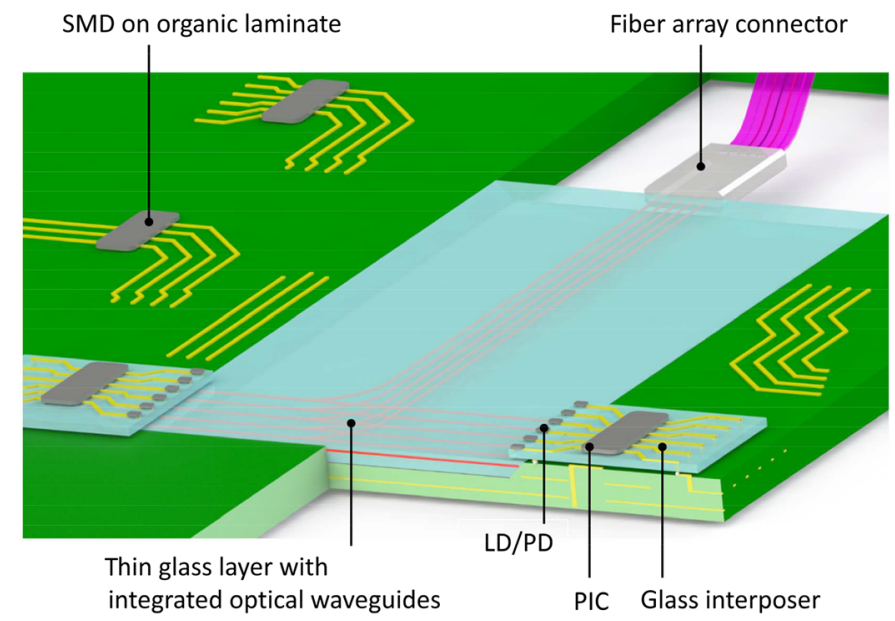

Fig. 2 Thin glass layer concept. Schematic drawing of EOCB cross-section with integrated optical waveguides buried in a thin glass layer which have been embedded in organic laminate, glass interposer with optical and electrical redistribution layer (RDL) for signal routing vertically through the different substrates, and assembled PIC, electronic, and optoelectronic components (LD and $\mathrm{PD}$ at the edge of the interposer).

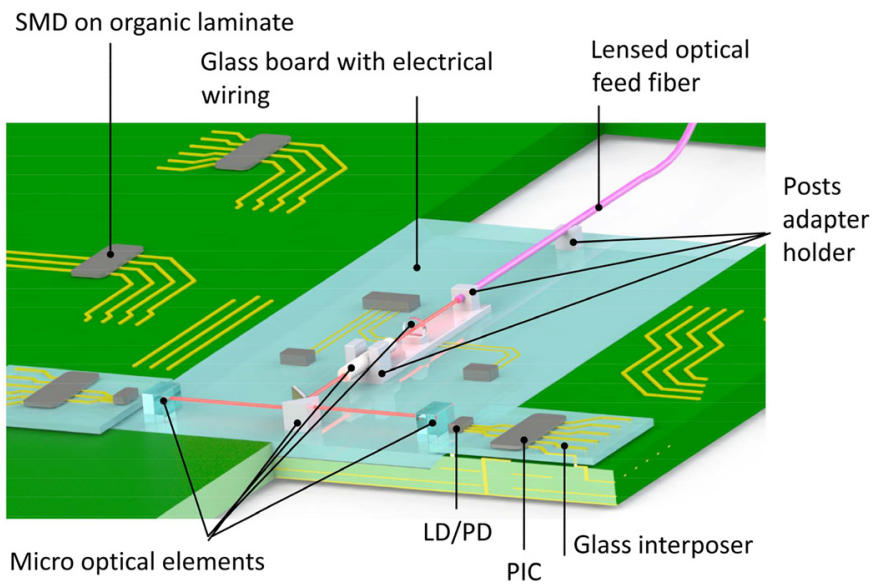

Fig. 3 Glass board concept. Schematic drawing of PCB with cross-section and optical subassemblies consisting of embedded bench-like glass board carrying optoelectronic and microoptic element assemblies, providing high optical alignment accuracy and stability, glass interposer with electrical RDL, and assembled PIC/electronic/optoelectronic components and LD/PD at the edge of the interposer. 
of the used glass substrates, we distinguish the thin glass layer concept from the glass board concept. The state-of-the-art will be elucidated, and higher level integration into printed circuit board (PCB) will be discussed, as well as several novel integration approaches on this material basis developed in our labs.

\section{Glass Integration Platform}

Glass is an ancient material and available in many different forms. It is widely utilized in conventional electronic and optoelectronic packaging, including as hermetic sealing for tungsten filament emitters or electron tubes. Various techniques for joining glasses and glassy materials have also been widely used, either by means of direct fusing (plasma and laser processing, anodic bonding) or mediated using glass solders or a variety of epoxies. However, only with the advent of very thin float glass production and the development of down draw processing for display applications, with precisely controlled thicknesses of typically $150 \mu \mathrm{m}$, did glass become a planar substrate material for electronic and photonic packaging at wafer and panel level. A selection of the most important properties is summarized in Table 1, including why these properties of glass are advantageous for electronic and photonic platforms.

As glass shows superior material properties, process ability and integration capabilities of glass will be the focus of this article. Glass has several advantages over other widely used substrate materials, such as silicon and organic materials. ${ }^{14-16}$ The specific dielectric properties of glass provide a high integration potential by combining electrical and optical functionalities in a single substrate. Large panel glass fabrication has grown tremendously in size and volume, driven by the success and wide-spread use of solar energy, as well as the development of thin film display panels. Despite being very thin, panels, as well as the machinery to handle and structure them, are continuing to increase in size and decrease in cost. The composition and tempering of glass, to increase its robustness, e.g., in mobile electronic devices, have also progressed remarkably.

The main disadvantage of glass for optoelectronic and photonic integration is its poor thermal conductivity. In particular, thermal management in components with high power dissipation requires much additional effort. In most cases, the solution is using heat spreading metal layers or thermal vias by means of the same processes as for electrical wiring and through glass vias (TGV), as discussed in Sec. 3.2.

For packaging purposes, the availability of suitable techniques for structuring and bonding are crucial and material related, respectively. Continuing challenges for glass are free-form cutting and cleaving, but the many advantages of laser technology $\left(\mathrm{CO}_{2}\right.$, short pulse laser, and combination with wet chemical etching) are increasingly used in fabrication on wafer and panel level. However, to some extent, the more traditional techniques of dicing and polishing remain important. In addition to the conventional bonding techniques of glass to glass or to other materials, such as anodic bonding, gluing, welding such as in MEMS and MOEMS, fabrication laser processing is being used more and more. ${ }^{17}$

For next generation multi-chip-module (MCM) integration, components such as electrical chips and optoelectronic PICs have to be assembled on platforms with both electrical and optical coupling and redistribution. Such electrical-optical platforms, called interposers, will need 3D optical single-mode links for PIC-specific wavelengths, in addition to electrical wiring and vias for chip-to-chip interconnects. Furthermore, they must conform to PCB mounting standards, such as ball grid array or land grid array, and appropriate optical interfaces to the optical PCB, also commonly referred to as electrical-optical circuit board (EOCB).

According the thickness of the used glass substrates, we distinguish the thin glass layer concept (sub-mm range) from the glass board concept ( $\mathrm{mm}$ range), which is described in the following.

The photonic interposer and EOCB integration approaches outlined above follow electronic packaging strategies for PCB and have to fit into their high-volume-manufacturing lines for panel size formats. Figure 2 depicts technical building blocks, with passive optical waveguides buried in a thin glass layer laminated into an EOCB, which can be connected to external optical fibers via array connectors, and glass-based interposer carrying PICs and e/o components, i.e., switches, LD, and PD. 


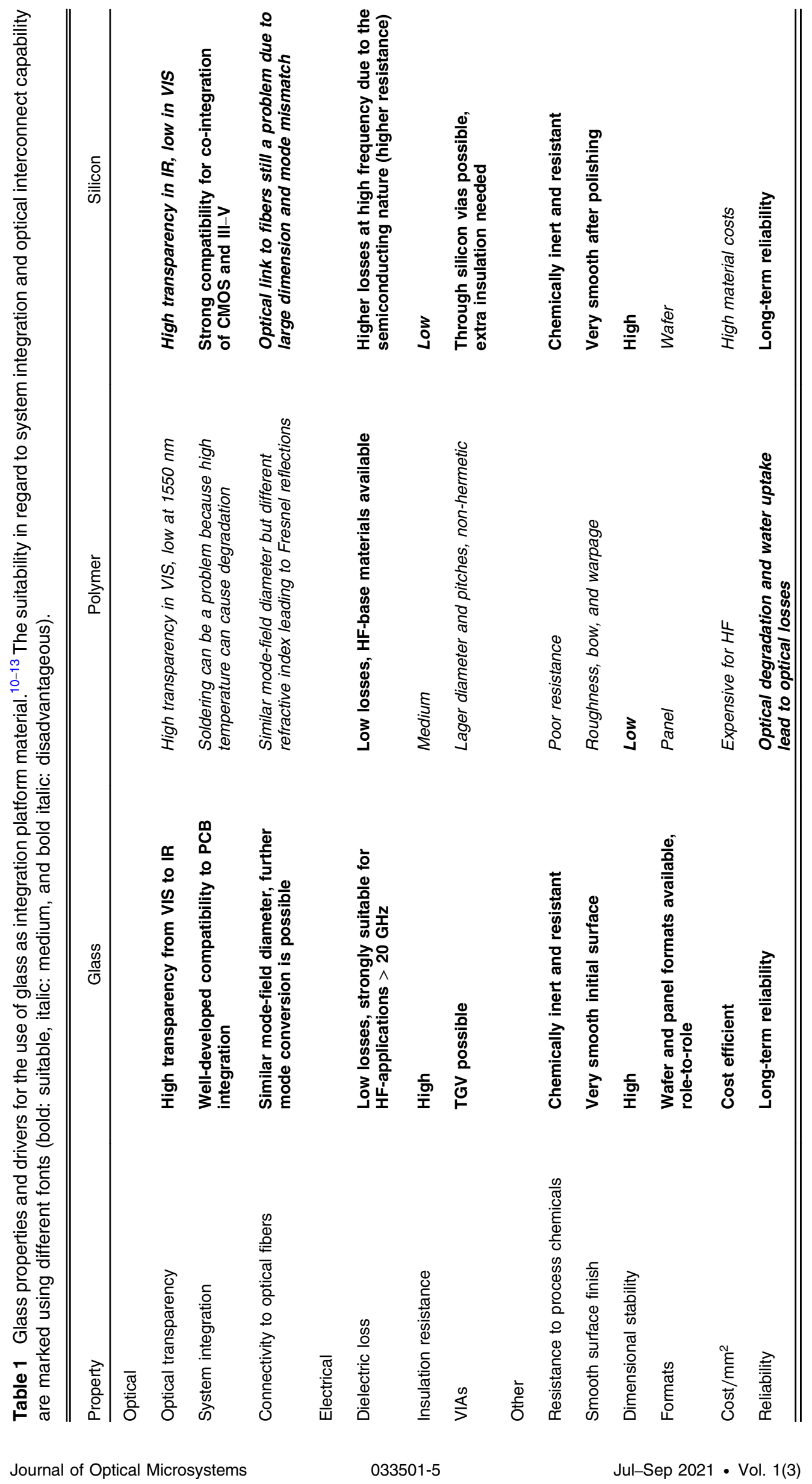


Thin glass (sub-mm range) as interposer material will facilitate thinner consumer products, communication and compute devices with increased bandwidth and performance, and high integration in photonic sensors.

Using thicker glass (mm-range), highly stable and miniaturized optical systems on glass boards can be defined - either standalone or embedded into a PCB environment, with or without integrated optical waveguides. Such glass boards host optical and electrical signal distribution, free-space optical elements, transparent windows with or without additional beam shaping optics, and hermetically sealed capping and interfaces to optical fiber interconnects. To create such complex systems, photonic design for packaging, advanced glass structuring technologies, and electronic packaging are merged with high-precision assembly. Figure 3 shows a schematic similar to Fig. 2 but of launching and splitting of a free-space beam from a lensed optical feed fiber. Alternatively, laser diodes or optical chips can get mounted onto the glass board directly or via structured holders. Glass interposers can interconnect PICs and other o/e components with PCB circuitry and optical functions on the glass board. Its size can be scaled from a few to tens of $\mathrm{cm}$ and be hermetically closed by additional glass frames and lids, not pictured in Fig. 3 .

A basic technical approach for the structuring of glass is laser technology. For the described platform (both concepts), we use $\mathrm{CO}_{2}$-laser scribing and short pulse laser ablation, in combination with wet chemical etching and tempering processes where needed to decrease roughness and enhance reliability. Optical grade surfaces, curved cavities, TGV, and channels can be realized this way, with a scaling potential on large-panel-manufacturing equipment as used in the display or solar panel industry.

Apart from photonic substrate integration strategies described above, optical fiber technology remains crucial. Optical fibers are commonly used for the glass integration platform to optically interconnect the subsystem with the periphery and for inter-system links, or to provide the sensor or data signals in remote systems. Connectors allow pluggability or need to be fixed permanently. Furthermore, extensive direct fiber-to-chip coupling schemes using mode field and/or pitch converters by adhesive gluing or direct welding exist and are well described. Glass-to-glass welding in particular is a one method suitable for the platform concept and is described in more detail below.

A great benefit has also been demonstrated by employing optical fibers from the interconnections even as sensors. ${ }^{18}$ This is closely related to the unique features exhibited by fiber optic sensors, such as high sensitivity, electromagnetic immunity, robustness, flexibility, compactness, and multiplexing. In this paper, we propose a very specific packaging for optical microresonators ${ }^{19}$ which are a class of resonant structures that offer an additional high degree of compactness and higher sensitivities in comparison to other well-known fiber optic sensors. This is due to their ability to strongly confine light at the interface of the resonator where a significant interaction takes place with the surrounding environment. However, they have a fundamental limitation due to the lack of integration technologies to integrate them into a practical and functional package. We show a very promising approach extending the thin glass platform to the third dimension in order to elucidate the advantages of our platform regarding to advanced fiber integration challenges such as for micro-bottle resonators besides common fiber-to-chip coupling.

The glass integration platform comprises a variety of electronic and photonic packaging features made possible by certain technologies discussed in the following sections in more detail. The main purpose of the platform is to interconnect hybrid optoelectronic, photonic, and microoptical components, and enable system integration in a very compact manner. The two main concepts as depicted schematically in Figs. 2 and 3 and are explained below by discussing some special demonstrators. Optical fiber interconnection technologies play a crucial role in all aspects but in particular for optical bottle resonator applications as described at the end of the paper.

\section{Thin Glass Layer Concept for Electrical-Optical Panel Integration}

The approach invented by Fraunhofer IZM in $1999^{20}$ to integrate an optical layer into a PCB has since been developed further by numerous research groups. The combination of electrical circuits and optical waveguides in one circuit board enables high-speed signal transmission and a high density of integration. In most cases, an optical layer based on glass is laminated into a 


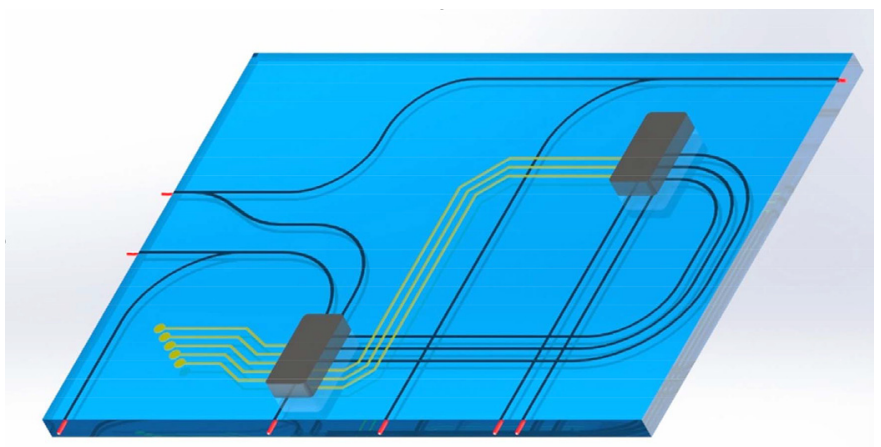

Fig. 4 Schematic drawing of a thin glass layer containing optical waveguides (black) and electrical wiring (yellow) on the top surface.

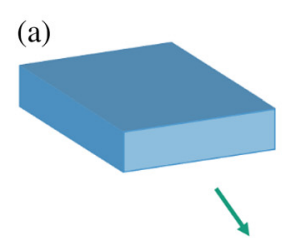

(b) (c)

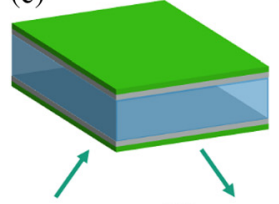

(d)

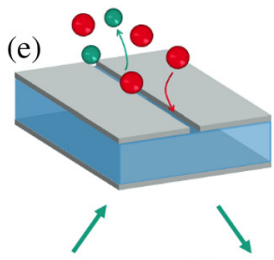

(f)

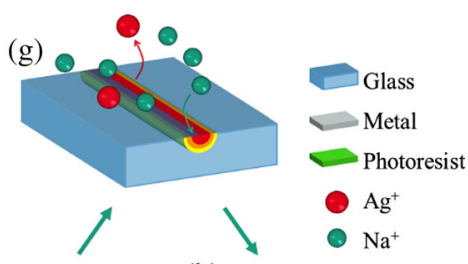

(h)
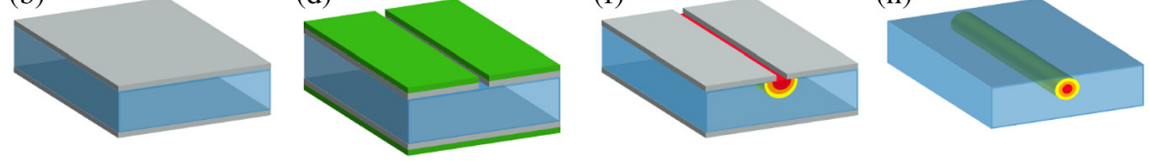

Fig. 5 lon-exchange process steps in thin display glass.

standard circuit board. However, the excellent properties of glass in terms of dimension stability, electrical high frequency, and a significantly increasing mechanical strength also allow EOCB to be fully made of glass.

As shown in Fig. 4, optical waveguides are integrated into the glass (as also depicted in Fig. 2), but additionally to Fig. 2 electrical circuits are applied and both optical and electrical components are placed on the board. Such platforms can be realized in single- and double-sided configurations. The waveguide fabrication process is described in the following section and Fig. 5.

The application-dependent build-up features the flexibility and diversity known from PCB manufacturing. Symmetrical approaches with one layer only have been developed, ${ }^{16}$ but also glass layers with two optical layers on bottom and top side, as well as multilayer boards of stacked or large horizontal glass layer design. ${ }^{21,22}$

\subsection{Glass Integrated Waveguides}

According to the requirements of data communications, the optical waveguides need to have low propagation loss of $<0.05 \mathrm{~dB} / \mathrm{cm}$ at 1310 and $1550 \mathrm{~nm}$, must operate in both single- and multimode, and should have a gradient index profile for low signal dispersion. Waveguides that can fulfill these requirements can be manufactured using an ion-exchange process, in which silver ions replace sodium ions and thus provide an increase in refractive index. The fabrication process is shown in Fig. 5. In detail, a metal layer is deposited on the glass (b) and then structured via photolithography using laser direct imaging (c)-(d). The masked glass is dipped into molten salt containing silver ions, which create a gradient index profile inside the glass in the unmasked areas (e)-(f). After removing the mask, a reverse ion-exchange buries the waveguides inside the glass (g)-(h). Based on this procedure, we developed a process running on commercial equipment, consisting of a $\mathrm{CO}_{2}$-laser cutting system, a sputtering system, a dip-coating system using a positive resist, and a laser direct imaging system. 
After starting the development on wafer level ${ }^{23}$ using wafer sized glasses and a mask exposure for lithography, the process was first scaled up to $297 \mathrm{~mm} \times 210 \mathrm{~mm}$ rectangular sized glass-panel with a flexible layout of the waveguides using laser direct imaging for lithography. ${ }^{24}$ Based on this, the size of the processable panel was increased to $457 \mathrm{~mm} \times 305 \mathrm{~mm} .{ }^{22} \mathrm{In} 2015$, graded-index high-bandwidth single-mode waveguides were shown using this process for the first time ${ }^{25}$ and most recently 2 -m-long spiral-shaped waveguides were demonstrated. ${ }^{26}$ Commercially available D $263^{\circledR} \mathrm{T}$ eco with a thickness of $550 \mu \mathrm{m}$ was used for the latter. Due to the low propagation losses of $0.059 \mathrm{~dB} / \mathrm{cm}^{26}$ and high process homogeneity, integrated functions can be focused in the future. On wafer level and on laboratory scale, multiple functions could be presented, ${ }^{27}$ with just one of many examples being direction coupler ${ }^{28}$ Y-junctions. ${ }^{29}$ In recent years, sensing applications using quantum dots ${ }^{30,31}$ and surface plasmon polaritons ${ }^{32}$ were also developed. A wide variety of chemically strengthened glasses with thicknesses down to $30 \mu \mathrm{m}$ have been developed for the display market. The suitability of such flexible glasses for optical applications has already been demonstrated. ${ }^{33,34}$ By improving glass quality and scaling up the processes for manufacturing, the optical waveguides for many of the applications proven in the laboratory setting can now be conceivably used in products. For most, a metallization of the glass is necessary.

\subsection{Metallization on Glass}

Metallization on glass can fulfill multiple functions, including fiducial marks, labeling, electrical circuits, and pads for wire bonding. The purpose determines the requirements for the material and the layer thicknesses. Building on research on the creation of diffusion mask for fabrication of optical waveguides with high adhesion and highly resolved structures in the range of $3 \mu \mathrm{m}$ mask opening width for single-mode waveguides, a wide range of different materials, and material combinations have been developed. For fiducial marks using a sputtering system, aluminum, chromium, copper, titanium, and tungsten-titanium can be deposited and structured on large panel sized glass, several of which are shown in Fig. 6. For electrical circuits with low current densities, the metals (e.g., copper) can be deposited with a thickness of up to $2 \mu \mathrm{m}$. Electrical circuits for higher current applications can be built up from sputtered seed layer and a sputtered start metallization such as copper, which can be thickened up to $30 \mu \mathrm{m}$ using galvanic plating.

For soldering and bonding processes, materials such as silver or gold are needed. Therefore, these were also applied to glass, as can be seen in Fig. 6(b), in which a gold metallization was applied via direct immersion. Electroless gold plating of copper resulted in 150-nm-thick solderable gold layers.

The combination of metallization and optical functions (see also Figs. 2 and 3) enables multiple functions and builds up a platform that serves multiple applications in the fields of data communications, sensing, and even quantum technologies. Fully embedded glass layers in organic PCB base materials allow high-speed line cards or optical backplanes as demonstrated in Fig. 7.

The electrical package made of multiple copper and FR4 prepreg layers can be fabricated using standard PCB technologies, which facilitates advanced high-density electrical PCB designs with multiple layers. A specific low-temperature lamination technique was developed
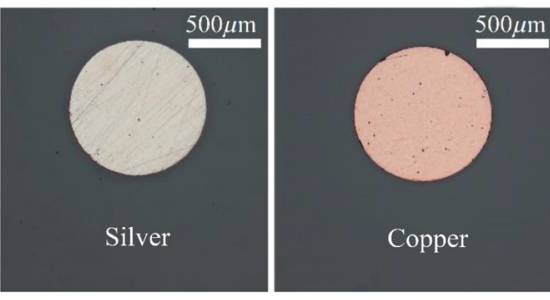

(a)
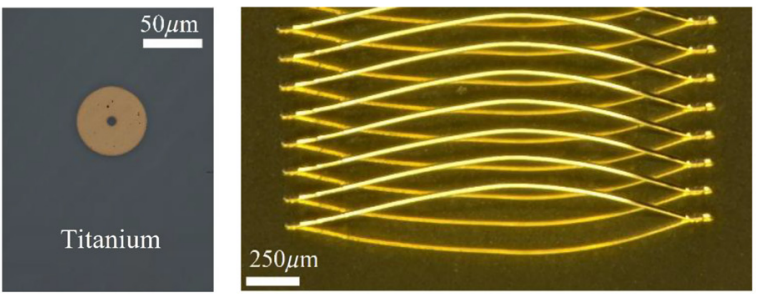

(b)

Fig. 6 (a) Fiducial marks on glass, made of silver, copper, and titanium. (b) Wire bonds on a gold metallization on glass. 


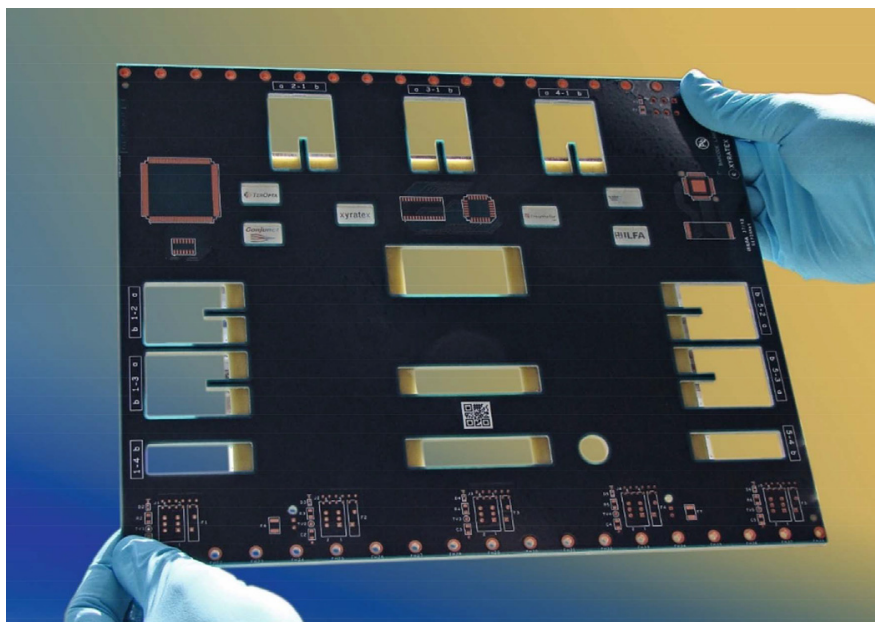

Fig. 7 EOCB demonstrator showing an optical backplane..$^{35}$

for bonding of the electrical cores to the inner optical layers. Applying the adhesive foils to the electrical core, one or multiple glass waveguide panels or double-side waveguide layer panels were set within a FR4 prepreg. Milled cut-outs for optical connector openings and cavities for optical engines $^{35}$ were milled using standard PCB equipment.

This basically two-dimensional platform can be extended to include the third dimension, as shown in the next section.

\section{Glass Board Concept for Photonic Free-Space Assemblies}

Increasingly complex assemblies of diverse components with various functionalities and geometries have been developed on basis of above described platform, using layouts, a generic form of which is shown in Fig. 3. Multiple optical, electronic, and mechanical interfaces can be designed and lasered into these boards, as will be elucidated in more detail below. Thin glass holding elements, adapters, and posts are structured to not only align various micro-optical or electrical-optical components to the optical axis but to also include visual and handling features for automated assembly by alignment machines. ${ }^{36}$ Especially, aspherical or free-form optical components often have to be aligned with all 6 degrees of freedom, often with precisions of 50 to $500 \mathrm{~nm}$ and a few arc seconds.

Thin glass structured by laser processes is used to improve adaption and positioning of various components such as light sources, beam shaping, and splitting components. Such posts define one or more optical planes above a mechanical reference plane nd can either be directly placed on a glass optical board or pre-assembled in separate alignment and joining processes, for which examples are given in Sec. 4.1.

Figure 8 shows an example of a passively and actively aligned optical system. Component and alignment accuracies are in the 1 to $20 \mu \mathrm{m}$ range depending on the intended optical performance in free-space beams that are split, coupled, and combined on the optical board. Active alignment is applied where needed for highest quality free-space beam alignment down to diffraction limits, or for adjustment to remaining beam divergence, shifting Gaussian beam positions to required positions, and single-mode coupling to optical components facets. The mechanical and electrical features for mounting electro-optical modulators and the two photo-detecting glass boards on the right side with optoelectrical conversion are mounted onto the main board (see Sec. 4.2 for more details). Visual features used for machine vision, recognition, and relative placing of components within micron accuracies are included in the assembly process of the glass board.

As mentioned above, highest quality optical alignment and coupling efficiencies typically require sub-micron machine accuracies, with light sources and detectors operated actively. This component light directly feeds back into the machine actor motion routines, transferring translational and rotational placing accuracies of machine actors into the assembled optical system. 


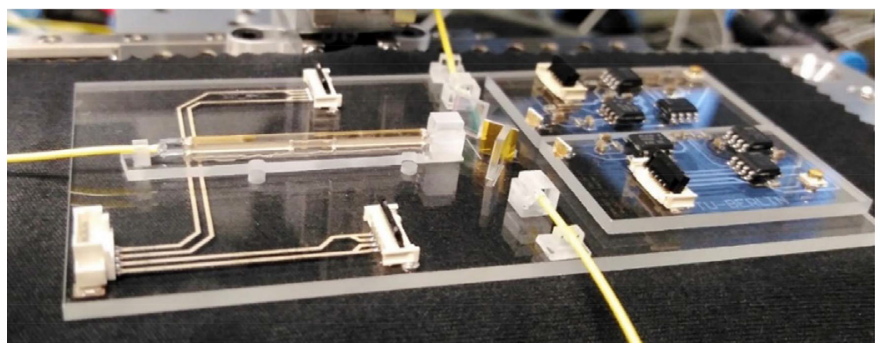

Fig. 8 Glass main board hosting a complex optical assembly on a $100 \mathrm{~mm} \times 50 \mathrm{~mm}$ footprint. A fiber-coupled external tunable laser is coupled on the left edge of the main board and is coupled into a separately mounted modulator, with its exit split and deflected/coupled into optical fibers, one at each on the long edges of the main board. These connect and bi-directionally read out an optically resonant external micro-bottle-resonator as described in Sec. 5 of this paper. Optical detection takes place on two separate glass boards mounted on the right.

Given the high cost of such finely aligning actors in industrial machines, usually only one is available per machine, requiring sequential alignments processes. Many-actor alignment machines for the alignment of optical systems are yet to be introduced. ${ }^{37}$ For even higher alignment accuracies, scanning of optical facets, and beam characterization, techniques currently found in laboratory settings are needed, such as piezo controlled hexapod systems, which achieve accuracies of 5 to $50 \mathrm{~nm}$ in $x / y / z$ and 1 to 5 arc sec in rotation.

\subsection{Preassembly of Free-Space Couplers}

As shown above, various optical components can be adapted and actively placed on an optical board based on laser structured glass. One or more precisely controlled optical planes above a mechanical plane can be defined by these means. Moreover, all degrees of freedom can be selected that are needed for later sequential alignment, ideally laterally (two directions) and rotationally (one axis). This allows separate and cost-effective preassembly, as pictured in Fig. 9 with a lens holder for forming a well-defined free-space beam from an optical feed fiber. Increasingly narrow beams can no longer be simply collimated and have to be treated and optically characterized as Gaussian beams, with specifically controlled beam waist positions, intensity, and amplitude/phase distribution: Highest free-space beam qualities and coupling efficiencies are ensured this way.

Controlling the Gaussian waist quality and position, creating focusing elements limited by diffraction alone is possible. By proper lens selection and holder design, a matching and aligning to almost any geometry/size of coupling spots on optical chips can be facilitated.

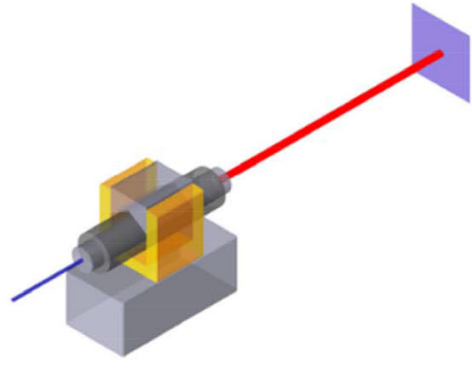

(a)

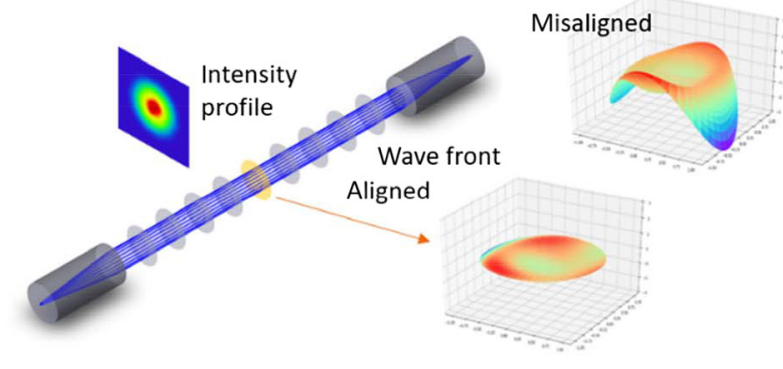

(b)

Fig. 9 (a) Optical component adaptor for a fiber-free-space collimation using lasered holding structures to be preassembled, with optical rays pictures. The actual simulation and characterization must resolve Gaussian beam properties. (b) Symmetrical free-space beam with centered Gaussian waist, in which a flat wavefront is ensured-typically by measurement using a ShackHartmann sensor. 
Here, we propose stacked glass block technology in combination with a fine alignment approach in the sub-micron range. This innovative puzzle technology features multiple degrees of freedom during the assembly of glass parts, compensating for undesired misalignments in coupling light to PIC's nano-waveguides. Moreover, adhesive-based issues can be reduced by stacking the glass parts with almost zero gap. The design of the glass fiber connector can easily be adapted to one or an array of single-mode optical fibers while its manufacturing from a glass panel is carried out. The potential use of fiber-based glass connectors has been recently demonstrated for two PIC-based modules, where light at a wavelength of $1550 \mathrm{~nm}$ is launched through a single input fiber (Fig. 10) and an array of input fibers (Fig. 11).

In Fig. 10, one of our standard adaptors is shown, consisting of three lasered glass parts that allow preassembling the fiber ferrule in the middle and performing the final alignment and fixing by means of the middle part, using active beam signal control on automated assembly machines.

The conceptual design in Fig. 10(a) consists of a four-piece glass connector, a PIC, an aluminum base, and a relief structure for the single input fiber. This practical and simple configuration meets the fundamental requirement of having an adhesive-free fiber interconnection. In particular, the stacked glass design for fiber-PIC coupling was proposed such that four glass pieces were used to further increase alignment tolerances and keep shrinkage effects down. To assure low-loss coupling, the PIC also contains integrated SSC where, as an optimal case, they allow reducing the insertion loss of the photonic module by as much as a factor of 3 . The assembly procedure is then defined as follows. The PIC was passively fixed to the base using a UV curable adhesive. To create the glass connector, a cleaved fiber was placed and adjusted inside a ferrule. The ferrule was also attached to an inner glass piece for better handling. Separately, three outer pieces were glued to support the mechanical stabilization of the outer part of the ferrule. Both parts were used for proceeding with the fiber-chip alignment and finally

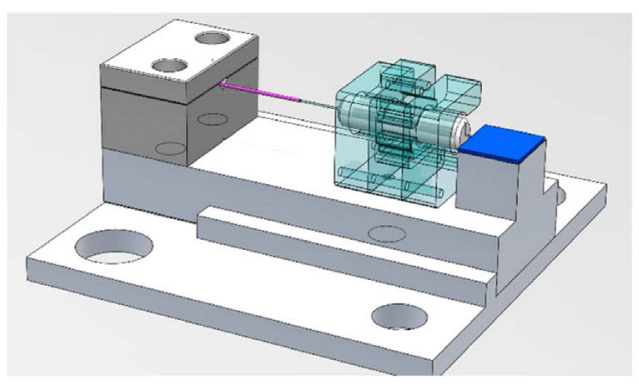

(a)

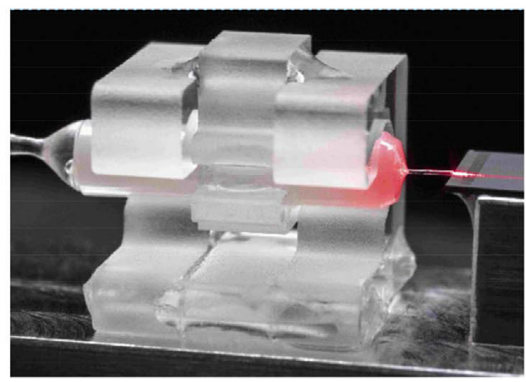

(b)

Fig. 10 Stacked glass adaptor for edge coupling of PICs: (a) CAD design of the hybrid photonic module and (b) functional assembled module with additional degrees of freedom on the middle segment part for lateral sub-micron control for coupling into the chip facet on the right side.

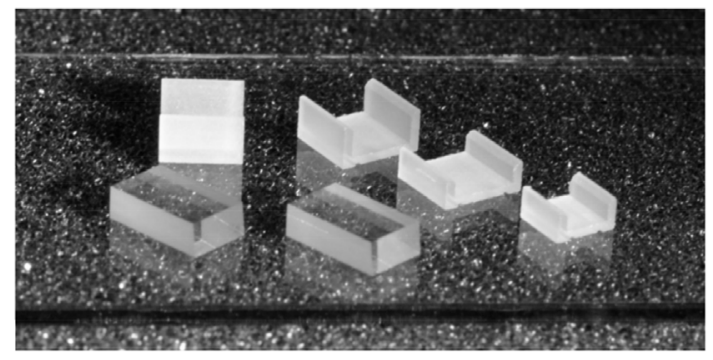

(a)

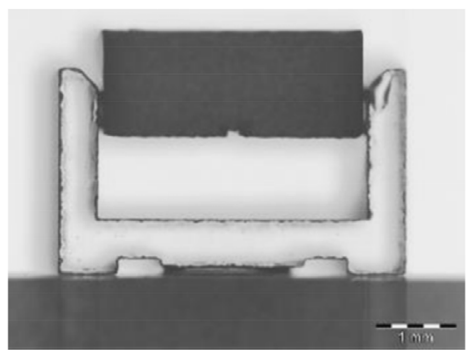

(b)

Fig. 11 Finely lasered adaptors for holding external converter chips used in coupling to PICs: (a) design for a 4-mm-long ion exchanged waveguide converter chip and (b) view of converter chip along optical waveguide axes, with waveguide plane glued into defined height in a lasered glass adaptor; on the base of the holder: gluing reservoirs/direct contacts for active alignment. 


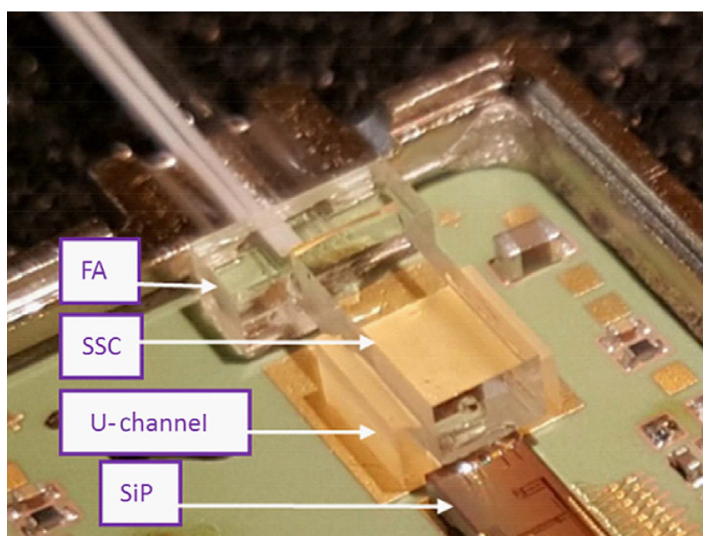

Fig. 12 Optical communication module with 4-fiber-to-PIC coupling by means of SSC in U-holder assembly.

glued together when the optimal value was achieved. After UV curing, an additional transmission loss of only about $0.3 \mathrm{~dB}$ was determined. Figure 10 (b) shows a photograph of the resulting fiber-based optical connector made from thin glass. The functionality of the packed PIC module was optically tested for about 2 months. It did not show significant optical degradation, which tells us that this technology is very promising for PIC modules that require free-space interconnections.

With regard to Fig. 11, the configuration of the U-holder is introduced to couple a 4-channel fiber array (FA) to optical waveguides of a silicon-on-insulator PIC as depicted in Fig. 12. The coupling loss of silicon nano-waveguides was reduced by introducing an external glass chip with SSC structures between the FA and the PIC. The commercially available SSC chip then reduces the insertion loss to the nano-waveguides from 6.87 to $0.5 \mathrm{~dB}$. The SSC was adhesively bonded to the PIC with the help of the U-holder. While UV-curing the adhesive, we observed a power loss of $1.3 \mathrm{~dB}$, which corresponds to transverse displacement of the SSC of $1.2 \mu \mathrm{m} .^{38}$

For the laser structuring of such holders, a fully automated 533-nm wavelength ns-lasersystem is used. All CAD designs for application specific holders, adapters, or posts can easily be modified. As large glass panels up to $600 \mathrm{~mm} \times 600 \mathrm{~mm}$ can be machined for structuring the parts of such holders, adapters, or posts, they can be manufactured at high volumes and it allows low cost and high reproducibility.

A very promising approach for fiber pig-tailing of glass interposers or SiN-PICs on glass substrate is a direct $\mathrm{CO}_{2}$-laser welding technique as developed ${ }^{39}$ and continuously improved to a high degree of automation. In contrast to common approaches using adhesive bonding, which have high challenges in terms of yield, reliability, and optical performance, this fiber welding technique for joining fused silica fibers on photonic substrates with integrated microoptics, e.g., waveguides, lenses, and lens arrays, is very reliable. It is a pure thermal process with a precise local heat transfer, which can combined with sequential passive or active alignment of each single fiber to the substrate allowing a high degree of flexibility and highest coupling efficiencies. Since the fiber is accessed only from one side, a two-dimensional high-density FA can be realized. The manufacturing time of such an interconnection is very short. Due to the adhesive-free interface, high power transmission is enabled, and the occurrence of polymer-caused misalignment and degradation is prevented.

\subsection{Optical Sub-Assemblies on Glass Boards}

Glass made boards can be stacked on each other to make optical sub-assemblies modular and able for pre-assembly on a glassy main board. Such miniaturized boards can serve as interposers to redistribute electrical wiring and interconnect systems on PCB and glass board as depicted in Fig. 2 or to carry special functional devices as part of a thicker and stiffer optical benchlike board concept, known as pre-assemblies, which can include micro-optic, optoelectronic, 


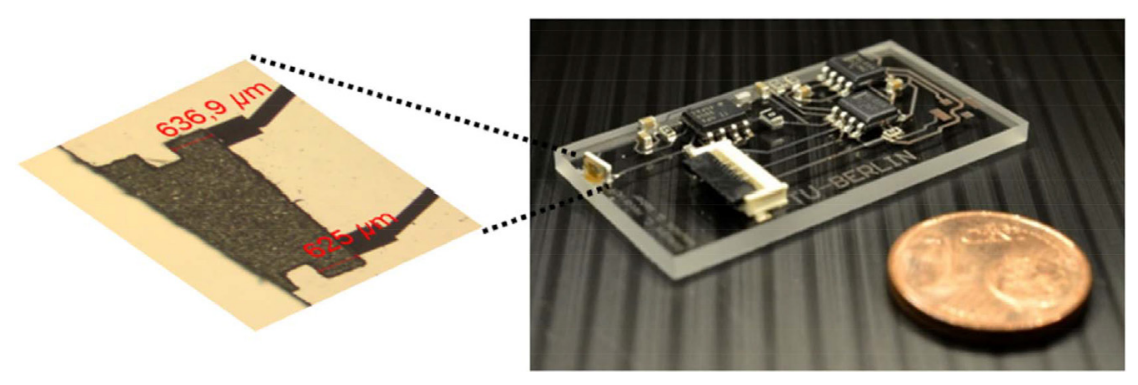

Fig. 13 A PD is mounted into a laser structured pocket (detail on left side) and wire bonded with electrical leads structured either by lithography or by laser decorating/stripping.

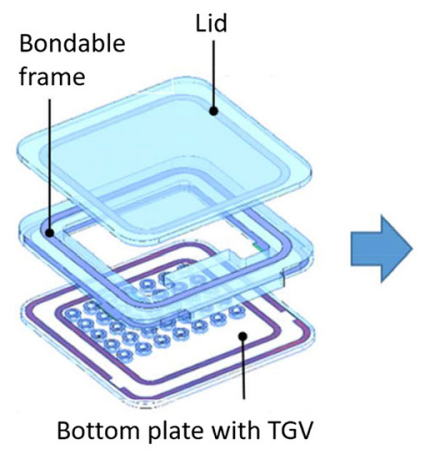

(a)

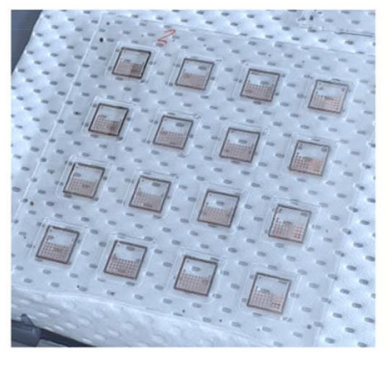

(b)

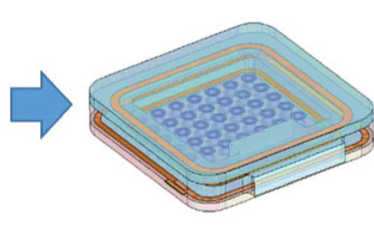

(c)

Fig. 14 (a) Concept of an optical multi-chip package and (b) photograph of a realized panel with 16 packages before singulation. (c) Design view of the singulated package w/o components.

or electronic components and connectors. Structured thin metallization on glass can be electrically bonded as described above in Sec. 3.2, enabling co-packaging of discrete and diverse electronics in the direct vicinity of optical sources or detectors. Figure 13 shows an example of a pre-assembled optoelectronic glass board for mounting onto a glassy main board (one of the two on the right side of the one depicted in Fig. 8) carrying a PD and discrete electronic amplifier chips placed and connected to features which are lasered both into glass and thin film metallization using processes compatible to surface mount technology.

Such pre-assembled glass boards can be rapidly processed and easily adapted to design modifications. Including electrical connectors, the board can be fully tested separately before mounting it on hosting main board.

Stacking of laser structured thin glass has much potential for cost-effective further development. The assemblies as introduced here can be sealed with glass frames and lids, creating compartments with optical windows for thermally isolated or hermetic operation. Here, the inherent low thermal conductivity and transparency of thin glass are advantages. Thermal and electrical through glass vias TGV can also be created where needed. ${ }^{40}$ In Fig. 14, a concept currently in development is shown, which extends the interposer concept to a multi-chip package made of glass using panel-level technologies. The structured base plate, frame, and lid can be laser structured and stacked into individual packages. Manufacturing is envisioned in panel scale production at lower cost.

\section{3D Integration of Optical Bottle Resonators for Sensing Applications}

Using the glass platform, we introduced the first robust package concept for optical sensors based on 3D fiber-based micro-bottle resonators, which exhibits extreme sensitivity (high $Q$ factors) and easier handling during assembly. Apart from their distinctive properties, they present further axial confinement of the light due to a harmonic effective potential deriving from the 


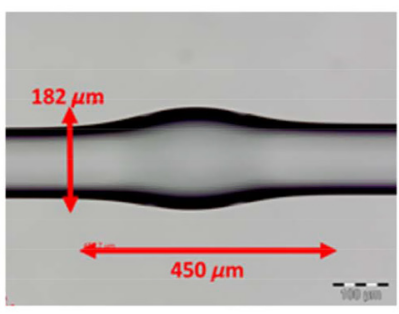

(a)

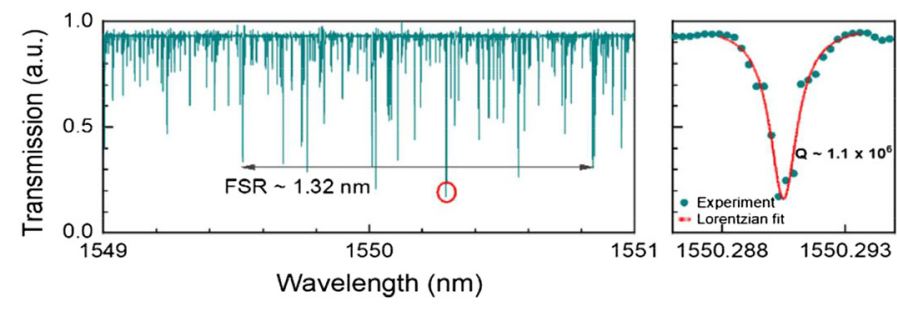

(b)

Fig. 15 (a) Fabricated glass micro-bottle resonator using a splicer and (b) its corresponding WGM spectrum measured with resolution of $0.1 \mathrm{pm}$. The red circle indicates the WGM analyzed by a Lorentzian fit where the resulting $Q$ value was found to be $\sim 1.1 \times 10^{6}$.

curvature of the resonator profile. This leads to the observation of a high density of optical modes, called whispering gallery modes (WGMs), which are indirectly measured by using the transmitted light of a tapered fiber. To understand the optical functionality of micro-bottles, a detailed study of WGMs has been previously reported. ${ }^{41}$ Glass micro-bottles are manufactured using standard single-mode fibers subjected to a soften-and-compress process. The tool used here is a commercial fiber splicer from Fujikura Ltd., specifically the model FSM100P+. The fiber was heated by arc discharge and repetitively compressed. The number of arc discharges was experimentally determined from 10 to 25 times with an arc duration of $1 \mathrm{~s}$. The parameters were also adjusted such that a parabolic-profile bottle can be guaranteed. The parabolic profile assures WGMs with high $Q$-factors $\left(10^{6}\right.$ to $\left.10^{7}\right)$. Several bottle-shaped fibers were then fabricated, with central diameters ranging from 180 to $250 \mu \mathrm{m}$ and bottle lengths between 300 and $400 \mu \mathrm{m}$. A microscope image of a fabricated glass micro-bottle with a diameter of $182 \mu \mathrm{m}$ and length of $450 \mu \mathrm{m}$ is shown in Fig. 15.

To produce adiabatic-profile tapered fiber, we employed a modular home-built fusion-andpull system. This includes three different heat sources $\left(\mathrm{CO}_{2}\right.$ laser, gas burner, and glass packaged infrared heater), allowing to develop several fiber fusion components made from glass, polymer, and chalcogenide glass. The tapered fiber for this work was prepared from a single-mode standard fiber using the gas burner as a heat source. The pendulum motion of the burner and the two pull stages were controlled in order to define a desired adiabatic profile with low-loss condition. The size of a standard single-mode fiber can then be reduced down to $3 \mu \mathrm{m}$. The explored process yielded tapered fibers with total lengths around $28 \mathrm{~mm}$ and a transmission efficiency of $94 \%$. To investigate the WGM spectrum in micro-bottles, light from a tunable laser with minimum step of $0.1 \mathrm{pm}$ was launched through the taper. The transmitted light was collected using a lownoise InGaAs photodetector. The scanned spectrum in Fig. 15 illustrates typical high density of notches (WGMs) from a parabolic-profile bottle. As expected, the Lorentzian fit of a notch yielded a $Q$-factor value of $2 \times 10^{6}$. The separation between two consecutives fundamental WGMs has been defined by the parameter free spectral range (FSR). With the analysis reported in Ref. 37, the FSR was determined to be $\sim 1.32 \mathrm{~nm}$.

After characterization of micro-bottles, a specific glass package solution was proposed. The glass package has a size of $10 \times 9 \times 54 \mathrm{~mm}^{3}$ and primarily comprising two protective parts (base and cover) and two holders that are perpendicular to each other as shown with the CAD design in Fig. 16(a). Using the laser-cutting system, the glass parts were structured from a thin glass panel. The micro-bottle and the taper were mounted and glued directly on their respective structured holder. The bottle holder was then placed tangentially on the top of the taper holder using an active alignment technique. After alignment, fixation of the bottle holder was performed by dispensing small drops of UV-curable adhesive. During the curing step, a fine alignment was repeated to assure good optical coupling. Finally, the glass cover in Fig. 16(b) was incorporated and sealed with the base containing the holders. The successful demonstration of packaged micro-bottles is illustrated in Fig. 16(c). Here, it is easy to see that the WGM spectrum is slightly different after assembly; however, the $Q$-factors of WGMs of $10^{6}$ can still be identified in the spectrum. Considering their use as gyroscopes, the sensitivity can be determined by tracking the rotation-related wavelength shifts of WGMs. If we keep the -factor of 


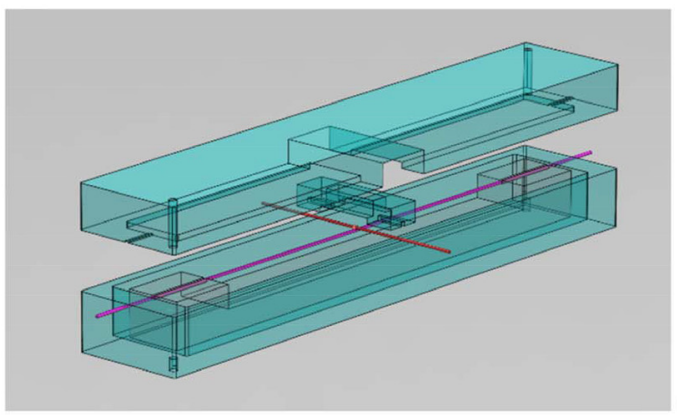

(a)

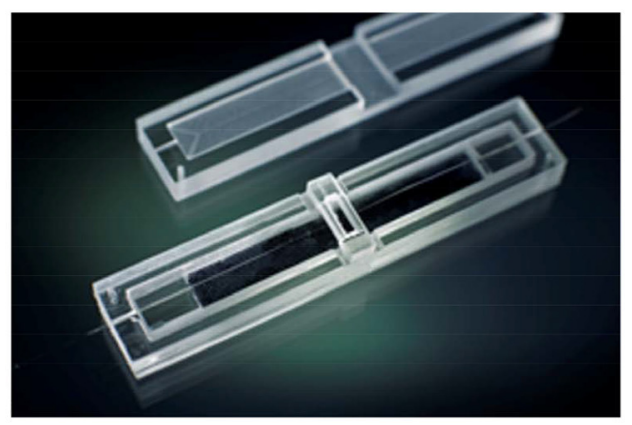

(b)

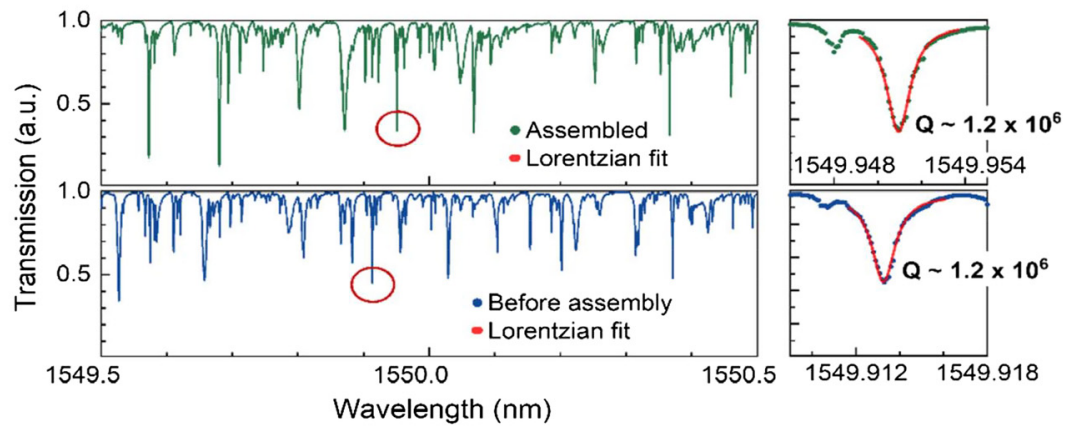

(c)

Fig. 16 Glass packaging for fiber-based micro-bottles: (a) CAD design and (b) fabricated glass package including the micro-bottle and the tapered fiber. (c) Spectra recorded with resolution of $0.1 \mathrm{pm}$ before and after assembly.

$10^{6}$ and increase the diameter of the resonator to more than $1 \mathrm{~mm}$, then a rotation rate value of $10^{\circ} / \mathrm{h}$ can be achieved.

\section{Summary}

This paper presents thin glass and specific packaging technologies for hybrid photonic system integration. Thin glass technology has many advantages, particularly in terms of cost-effectiveness, as well as dielectric and optical properties. Our review of the literature on glass material properties, processes, and applications not only shows that thin glass layers with integrated optical waveguides and the thin glass board with assembled components can be used to redress the longstanding underdevelopment of photonic packaging, but that additional optical and electrical functionality can be integrated by fiber coupling and metallization processes. Both concepts are feasible to be laminated and embedded into a PCB, respectively. Specific demonstrators have been shown: EOCB and a sensor platform. Fiber optical interconnects are discussed for PIC coupling by means of glass-made holding structures and with regard to micro-resonator packaging by means of specific 3D glass-made packages. All the processes can be applied on large panel level to enable large integrated systems. But the technology is also suitable for dicing for high-volume interposers or sensor platforms. The core technologies are ion exchange to integrate optical waveguides, metallization, structuring and assembly of specific holding elements and adapters, laser welding of glass fibers to glass, and micro-bottle resonator fabrication and packaging.

\section{Acknowledgments}

We thank all of our co-workers for wonderful experimental work and technical expertise. Furthermore we thank our sponsors for supporting this work in multiple projects, including "MiReG" IGF 19619N from German Federal Ministry of Economics and Technology; "HighConnect" 03VP00880 from German Federal Ministry of Education and Research; and "Chip SCG" 01QE1705C from Bundesministerium für Bildung und Forschung. 


\section{References}

1. E. Mounier, "Data center O/Is could pave the way for the photonic-in-package era," Chip Scale Rev. 1, 11-12 (2017).

2. D. Miller, "Device requirements for optical interconnects to silicon chips," Proc. IEEE 97(7), 1166-1185 (2009).

3. L. Alloatti, "High-speed photonics for side-by-side integration with billion transistor circuits in unmodified CMOS processes," J. Lightwave Technol. 35(6), 1168-1173 (2017).

4. A. Ghiasi, "Large data centers interconnect bottlenecks," Opt. Express 23(3), 2085-2090 (2015).

5. Teem Photonics, "WAFT waveguide interface products," https://www.teemphotonics.com/ integrated-optics/waft-interface-products/ (accessed 9 January 2021).

6. R. Oven, "Tapered waveguides produced by ion exchange in glass with a nonuniform electric field," J. Lightwave Technol. 24(11), 4337-4344 (2006).

7. D. K. Qing, K. Itoh, and M. Murabayashi, "Fabrication of spot size converters for optical chemical sensors by distributed-field-assisted ion-exchange," Opt. Rev. 3(2), 114-119 (1996).

8. N. Psaila, "3D laser direct writing for advanced photonic integration," Proc. SPIE 10924, 109240U (2019).

9. J. Liu et al., "Double inverse nanotapers for efficient light coupling to integrated photonic devices," Opt. Lett. 43(14), 3200-3203 (2018).

10. A. Pizzagalli, "Glass substrate for semiconductor applications: 2020 market and technology report," Yole Dévelopment.

11. S. Choi et al., "Signal integrity analysis of silicon/glass/organic interposers for 2.5D/3D interconnects," in IEEE 67th Electronic Components and Technology Conference (ECTC), pp. 2139-2144 (2017).

12. B. M. D. Sawyer et al., "Design and demonstration of a 2.5-D glass interposer BGA package for high bandwidth and low cost," IEEE Trans. Compon., Packag. Manufact. Technol. 7(4), 552-562 (2017).

13. A. Usman et al., "Interposer technologies for high-performance applications," IEEE Trans. Compon., Packag. Manufact. Technol. 7(6), 819-828 (2017).

14. M. Topper et al., "3-D thin film interposer based on TGV (through glass vias): an alternative to Si-interposer," in Proc. 60th Electron. Comp. and Technol. Conf., Las Vegas, Nevada, pp. 66-73 (2010).

15. L. Brusberg et al., "Glass carrier based packaging approach demonstrated on a parallel optoelectronic transceiver module for PCB assembling," in Proc. 60th Electron. Comp. and Technol. Conf., Las Vegas, Nevada, pp. 269-274 (2010).

16. M. Neitz et al., "Design and demonstration of a photonic integrated glass interposer for midboard-optical engines," in IEEE 67th Electron. Comp. and Technol. Conf., Orlando, Florida, pp. 538-544 (2017).

17. R. R. Gattass and E. Mazur, "Femtosecond laser micromachining in transparent materials," Nat. Photonics 2, 219-225 (2008).

18. B. Lee, "Review of the present status of optical fiber sensors," Opt. Fiber Technol. 9(2), 57-79 (2003).

19. J. Ward and O. Benson, "WGM microresonators: sensing, lasing and fundamental optics with microspheres," Laser Photonics Rev. 5(4), 553-570 (2011).

20. D. Krabe and W. Scheel, "Optical interconnects by hot embossing for module and PCB technology - the EOCB approach," in 49th Electron. Comp. and Technol. Conf., San Diego, California (1999).

21. H. Schroder et al., "Multi-layer electro-optical circuit board fabrication on large panel," in IEEE 66th Electron. Comp. and Technol. Conf., Las Vegas, Nevada, pp. $468-476$ (2016).

22. L. Brusberg et al., "Large optical backplane with embedded graded-index glass waveguides and fiber-flex termination," J. Lightwave Technol. 34(10), 2540-2551 (2016).

23. H. Schröder et al., "Planar glass waveguides for high performance electrical-optical-circuitboards (EOCB)—the glass-layer-concept," in 53rd Electron. Comp. and Technol. Conf., Proc., New Orleans, Louisiana, pp. 1053-1059 (2003). 
24. H. Schröder et al., "Glass panel processing for electrical and optical packaging," in 61st Electron. Comp. and Technol. Conf. (2011).

25. L. Brusberg et al., "Single-mode board-level interconnects for silicon photonics," in Opt. Fiber Commun. Conf. and Exhib., Los Angeles, California, pp. 1-3 (2015).

26. J. Schwietering et al., "Integrated optical single-mode waveguide structures in thin glass for flip-chip PIC assembly and fiber coupling," in 70th Electron. Comp. and Technol. Conf. (2020).

27. A. Tervonen, B. R. West, and S. Honkanen, "Ion-exchanged glass waveguide technology: a review," Opt. Eng. 50(7), 071107 (2011).

28. R. G. Walker and C. D. W. Wilkinson, "Integrated optical waveguiding structures made by silver ion-exchange in glass. 2: directional coupler and bends," Appl. Opt. 22(12), 1929-1936 (1983).

29. T. J. Cullen and C. D. W. Wilkinson, "Radiation losses from single-mode optical Y junctions formed by silver-ion exchange in glass," Opt. Lett. 9(4), 134-136 (1984).

30. X. Xu et al., "Towards the integration of nanoemitters by direct laser writing on optical glass waveguides," Photonics Res. 8(9), 1541 (2020).

31. J. B. Madrigal et al., "Hybrid integrated optical waveguides in glass for enhanced visible photoluminescence of nanoemitters," Appl. Opt. 55(36), 10263-10268 (2016).

32. R. Tellez-Limon et al., "Excitation of surface plasmon polaritons in a gold nanoslab on ion-exchanged waveguide technology," Appl. Opt. 59(2), 572-578 (2020).

33. L. Li et al., "A new twist on glass: a brittle material enabling flexible integrated photonics," Int. J. Appl. Glass Sci. 8(1), 61-68 (2017).

34. G. Macrelli, A. K. Varshneya, and J. C. Mauro, "Ultra-thin glass as a substrate for flexible photonics," Opt. Mater. 106, 109994 (2020)

35. H. Schröder et al., "Electro-optical backplane demonstrator with integrated multimode gradient-index thin glass waveguide panel," Proc. SPIE 9368, 93680U (2015).

36. W. Lewoczko-Adamczyk et al., "Thin glass based optical sub-assemblies for embedding in electronic systems," in IEEE 68th Electron. Comp. and Technol. Conf., San Diego, California, pp. 1136-1139 (2018).

37. S. Kibben, M. Seyfried, and T. Vahrenkamp, "Intelligent simultaneous active sub-micron multi-component alignment for next generation datacom applications," Proc. SPIE 10899, 1089909 (2019).

38. V. Z. Gómez et al., "Fiber-coupled photonic interconnects based on stacked glass block connectors," Proc. SPIE 10924, 109240W (2019).

39. H. Schröder et al., "Array fiber welding on micro optical glass substrates for chip-to-fiber coupling," Proc. SPIE 8991, 89910F (2014).

40. K. Kröhnert et al., "Reliabillity of through glass vias and hermetically sealing for a versatile sensor platform," in IEEE 8th Electron. Syst.-Integr. Technol. Conf., Tønsberg, Vestfold, pp. 1-6 (2020).

41. J. Herter et al., "Experimental demonstration of temperature sensing with packaged glass bottle microresonators," Sensors 18, 4321 (2018).

Henning Schröder is heading the Optical Interconnection Technologies Group at Fraunhofer IZM. He received his PhD from the Technical University of Berlin in 2000. He specializes in $\mathrm{R} \& \mathrm{D}$ of optical interconnection technologies for printed circuit boards (PCB) and photonic modules, with a focus on the design, fabrication, and performance enhancement of optical glass waveguides and micro-optics for PCB, and optical sensors, their characterization, and on reliable micro-optical assembling and packaging technologies including fiber optics. He holds numerous patents in photonic packaging technologies.

Julian Schwietering received his MSc degree in technical physics from the Technical University of Ilmenau, Germany, in 2018. Since then he has been working as a research assistant at Technical University of Berlin in the field of electro-optical circuit boards made of glass. In particular, he focuses on the ion-exchange process to integrate optical waveguides and other optical structures into glass. This includes experimental work as well as simulations. 
Gunnar Böttger currently is senior scientist at Fraunhofer IZM and responsible for automated photonic system assembly. He directs application oriented research projects, with a focus on novel opto-electronic packaging approaches using laser processed thin glass. Before joining Fraunhofer IZM in 2012, he worked in industry as a research manager. As a physicist he holds a PhD from Hamburg University of Technology in micro optics, and also was a postdoctoral researcher at Karlsruhe Institute of Technology.

Vanessa Zamora received her PhD in physics at the University of Valencia in 2010. She briefly worked at the Max Planck Institute for Polymer Research in Mainz, Germany. She was a postdoctoral fellow at the University of Alberta, Canada. Later, she joined the Fraunhofer HHI in Berlin. Currently she is a senior scientist at Fraunhofer IZM and Technical University of Berlin. Her research covers low-loss coupling solutions and packaging for integrated photonics and optical microresonators. 